\title{
BMJ Global Health Reducing maternal mortality in China in the era of the two-child policy
}

\author{
Jue Liu, ${ }^{1}$ Li Song, ${ }^{2}$ Jie Qiu, ${ }^{2}$ Wenzhan Jing, ${ }^{1}$ Liang Wang, ${ }^{2}$ Yue Dai, ${ }^{2}$ Geng Qin, ${ }^{2}$ \\ Min Liu (D) ${ }^{1}$
}

To cite: Liu J, Song L, Qiu J, et al. Reducing maternal mortality in China in the era of the two-child policy. BMJ Global Health 2020;5:e002157. doi:10.1136/ bmjgh-2019-002157

Handling editor Seye Abimbola

- Additional material is published online only. To view please visit the journal online (http://dx.doi.org/10.1136/ bmjgh-2019-002157).

$\mathrm{JL}$ and LS contributed equally., $\mathrm{GQ}$ and $\mathrm{ML}$ contributed equally.

Received 12 November 2019 Revised 12 December 2019 Accepted 22 December 2019

\section{Check for updates}

(C) Author(s) (or their employer(s)) 2020. Re-use permitted under CC BY-NC. No commercial re-use. See rights and permissions. Published by BMJ.

${ }^{1}$ Department of Epidemiology and Biostatistics, School of Public Health, Peking University Health Science Centre, Beijing, China

${ }^{2}$ Department of Maternal and Child Health, National Health Commission of the Peoples Republic of China, Beijing, China

\section{Correspondence to}

Professor Min Liu; liumin@bjmu.edu.cn and Dr Geng Qin;

qingeng@nhfpc.gov.cn

\section{ABSTRACT}

Reducing maternal mortality ratio (MMR) is of great concern worldwide. After the implementation of the twochild policy in 2013, the number of live births and the proportion of high-risk pregnancies both increased, and these bring new challenges to the reduction of MMR. China implemented a package of nationwide strategies in April 2016, the Five Strategies for Maternal and Newborn Safety (FSMNS). The FSMNS consists of five components: (1) pregnancy risk screening and assessment strategy, (2) case-by-case management strategy for high-risk pregnancies, (3) referral and treatment strategy for critically ill pregnant women and newborns, (4) reporting strategy for maternal deaths (and 5) accountability strategy. To better implement the FSMNS, China formulated a unified pregnancy risk screening form. After risk assessment and classification, medical records of all the pregnant women are labelled with green (low risk), yellow (moderate risk), orange (high risk), red (highest risk) or purple (infectious disease) for tailored management. By the implementation of FSMNS, China has already kept the MMR stable and cause it to enter a controlled decline. MMR in China has declined by $21.1 \%$, from 23.2 per 100000 live births in 2013 to 18.3 per 100000 live births in 2018. The country's challenges and experience in reducing the MMR could provide useful lessons for other countries.

\section{INTRODUCTION}

Reducing maternal mortality ratio (MMR) is of great concern worldwide. ${ }^{1}$ China, a developing country, has achieved the Millennial Development Goals on MMR ahead of schedule, and it has made remarkable progress in promoting maternal and child health. $^{2-4}$ As the world's most populous country, China accounts for about one-fifth ( 1.38 billion) of the world's population and is impactful to the achievement of global MMR reduction target (less than 70 per 100000 live births by 2030 at average) in the Sustainable Development Goals (SDGs). To continuously improve maternal and infant health in China, the Chinese government has set a new target of reducing the MMR to 12 per 100000 live births by 2030 in the Healthy China 2030

\section{Summary box}

An increased proportion of high-risk pregnant women poses a challenge to reducing the sustained maternal mortality ratio (MMR) in China in the two-child policy era.

- A package of strategies, the Five Strategies for Maternal and Newborn Safety, on better management of high-risk pregnant women has been implemented, and the MMR in China has remained stable and entered a controlled decline.

- Better management of high-risk pregnant women is crucial to MMR reduction.

- The Five Strategies for Maternal and Newborn Safety should be constantly implemented in China to achieve the targets of Sustainable Development Goals and Healthy China 2030 with respect to reducing maternal mortality by 2030 .

plan, based on the previous trends of MMR reduction in China. ${ }^{5}$

However, after the implementation of the two-child policy in 2013, the number of live births and the proportion of high-risk pregnancies both increased, and these bring new challenges to the goal of reducing the MMR in the Healthy China 2030 Plan. We here analyse the challenges inherent in reducing the MMR in the two-child policy era and the comprehensive strategies China has already implemented to keep the MMR stable and cause it to enter a controlled decline. The country's challenges and experience in reducing the MMR could provide useful lessons for other countries.

\section{CURRENT STATUS OF MMR REDUCTION IN CHINA}

In the past few decades, the MMR in China has declined by $46.5 \%$, from 34.2 per 100000 live births in 2008 to 18.3 per 100000 live births in $2018 .{ }^{4}$ Urban-rural and regional disparities in the MMR in China have shrunk (figure 1), and the MMR at country level is lower than the average of the Western Pacific Region (41 per 100000 live births).${ }^{67}$ Despite 

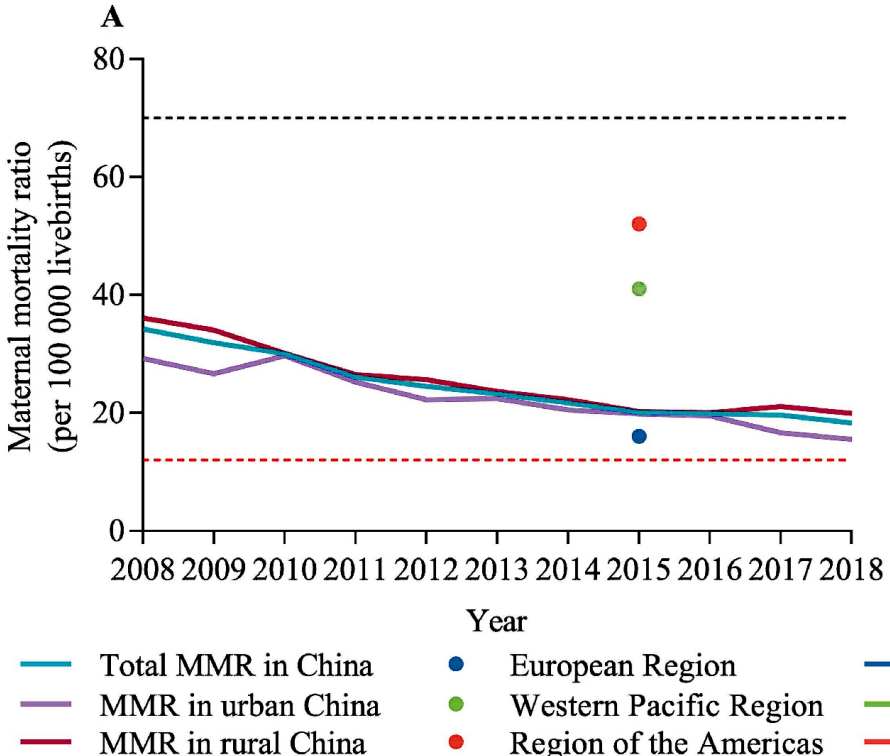

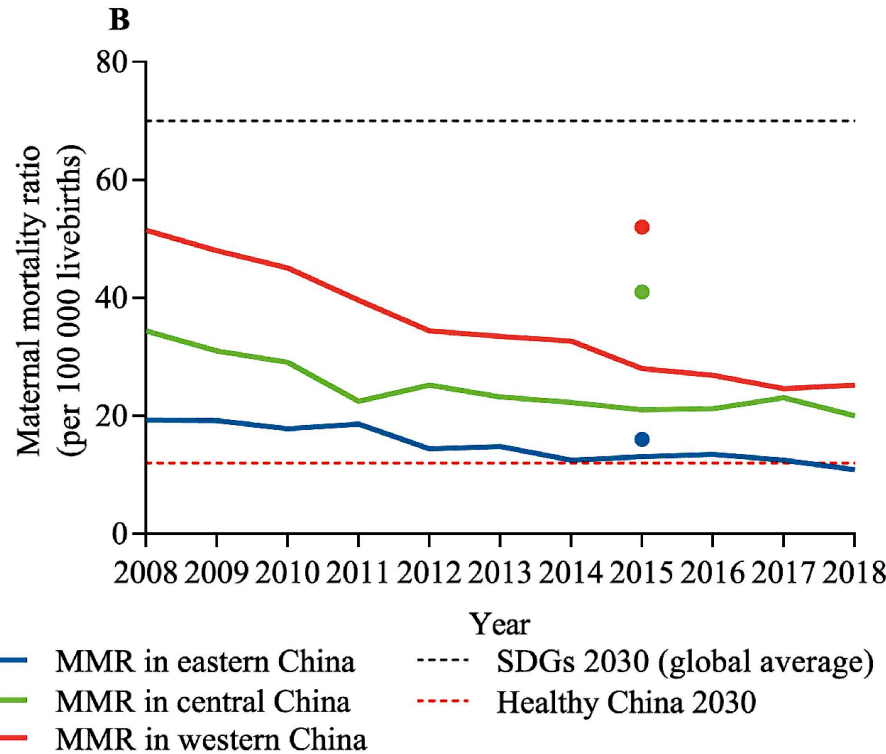

Figure 1 Maternal mortality ratio (MMR) by residency (A) and region (B) in China, 2008-2018. Notes: data were extracted from the National Health Statistics Yearbooks in China and World Health Statistics by WHO. ${ }^{47}$

the decline, the MMR in China is still higher than that of the WHO European Region (16 per 100000 live births) and lags behind the targets in the Healthy China 2030 Plan (12 per 100000 live births). ${ }^{67}$

\section{CHALLENGES TO SUSTAINED MMR REDUCTION IN THE TWO- CHILD POLICY ERA}

In November 2013, the Chinese government changed the one-child policy that had been in place for three decades to allow couples in which at least one of the marital partners was an only child to have a second child (selected two-child policy). In October 2015, the Chinese government announced that all couples would be allowed to have two children (universal two-child policy). ${ }^{8}$ After the implementation of the two-child policy, the proportion of high-risk pregnancies increased from $19.4 \%$ in 2013 to $24.7 \%$ in 2016, compared with $15.7 \%$ in 2008 (figure 2). ${ }^{469}$ It even exceeded $50 \%$ in some developed

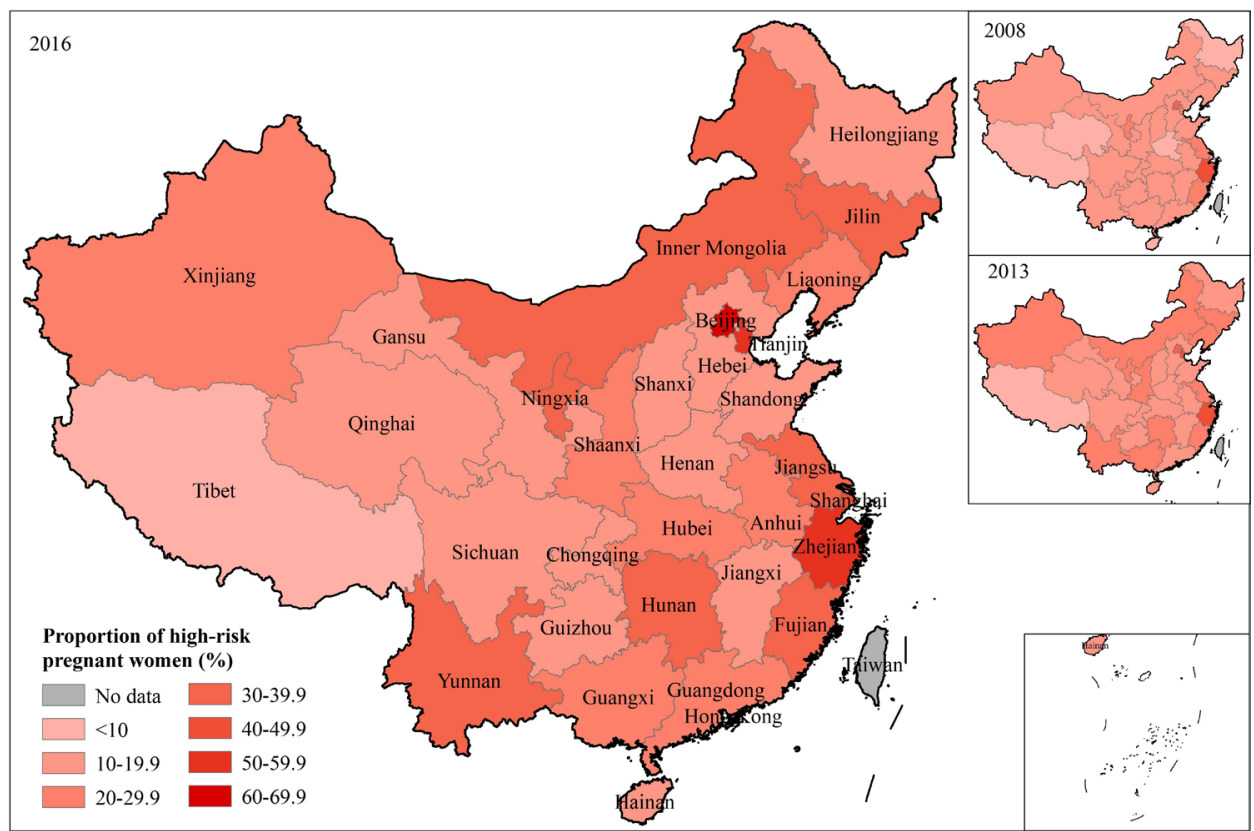

Figure 2 Proportion of high-risk pregnant women by province in 2008, 2013 and 2016. Notes: data were extracted from the National Health Statistics Yearbooks in China. The proportion of high-risk pregnant women is defined as the ratio of the number of high-risk pregnant women (any condition that could increase the likelihood of an adverse outcome for mother, fetus or both) to the number of live births. ${ }^{4}$ High-risk pregnant women have been further labelled with yellow (moderate risk), orange (high risk), red (highest risk) or purple (infectious disease) after the implementation of Five Strategies for Maternal and Newborn Safety since 2016. 


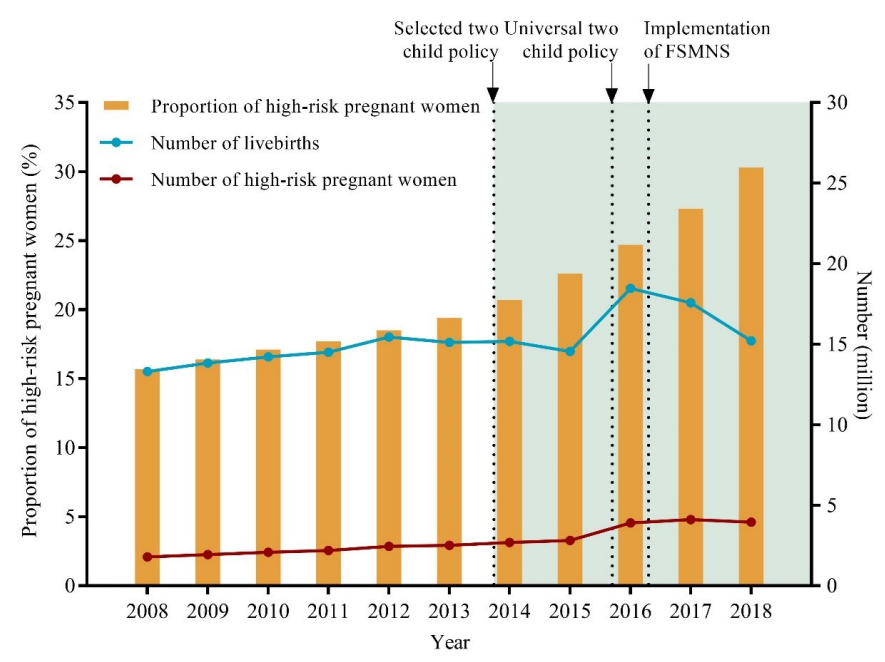

Figure 3 Proportion of high-risk pregnant women in China, 2008-2018. Notes: data were extracted from the National Health Statistics Yearbooks in China. ${ }^{4}$ FSMNS, the Five Strategies for Maternal and Newborn Safety.

eastern provinces. Along with the increase in overall live births in the two-child policy era, the number of high-risk pregnancies also increased significantly (figure 3 ). ${ }^{4}$

High-risk pregnancies are defined as any condition that could increase the likelihood of an adverse outcome for mother, fetus or both. ${ }^{9}$ Women with high-risk pregnancies are the vital portion of the population most likely to be subject to maternal death. Promptly identifying and effectively managing high-risk pregnancies is key to MMR reduction. Otherwise, there might be a rebound in the MMR and the targets of SDGs and Healthy China 2030 would be missed.

\section{CAUSES OF THE CHALLENGES}

The challenges to sustained MMR reduction have specific causes. First, the growing global prevalence of chronic conditions (such as obesity, diabetes, hypertension and heart disease) has increased the number of high-risk pregnant women with related complications. ${ }^{10} 11$ The MMR has rebounded in some developed countries, showing an upward trend. ${ }^{11}{ }^{12}$ Second, women are having children later in life than they used to. ${ }^{11}$ A large number of obstetric complications are associated with advanced maternal age. These include fetal anomalies, stillbirth, pre-eclampsia, gestational diabetes mellitus and so on. ${ }^{13}$ Third, because of the imbalance in socioeconomic development and disparities on the distribution of health resources, there is a corresponding conflict between limited health resources and increased demand for maternal and child health services. Countries are actively exploring how to cope with the increased number of high-risk pregnant women and reducing the MMR. ${ }^{13}$

In addition to the global challenges mentioned above, China has also faced some unique issues after the relaxation of the one-child policy. The proportion of multiparous pregnancies increased from $34.1 \%$ in 2012 to $46.7 \%$ in $2016 .{ }^{14}$ As a country with a relatively high rate of caesarean delivery previously, ${ }^{15}$ the proportion of pregnant women with uterine scars has correspondingly doubled from $9.8 \%$ in 2012 to $17.7 \%$ in $2016 .{ }^{14}$ Previous works have shown that the proportions of pregnant women with advanced maternal age, over 40 years, prevalence of gestational hypertension, gestational diabetes mellitus, dangerous placenta previa, placenta implantation, severe postpartum haemorrhage and other complications also increased after the relaxation of the one-child policy. ${ }^{16}{ }^{17}$ Although the four leading causes of maternal mortality remain obstetric haemorrhage, amniotic fluid embolism, pregnancy-induced hypertension and heart disease in China, the proportion of indirect (non-obstetric) causes of maternal mortality, such as heart disease, has increased (figure 4). MMR caused by heart disease has increased from 1.8 per 100000 live births in 2013 to 3.3 per 100000 live births in 2016. All of the reasons given here contribute to the significant increase in the proportion of high-risk pregnancies, which place heavy challenges on reducing maternal mortality in the two-child policy era.

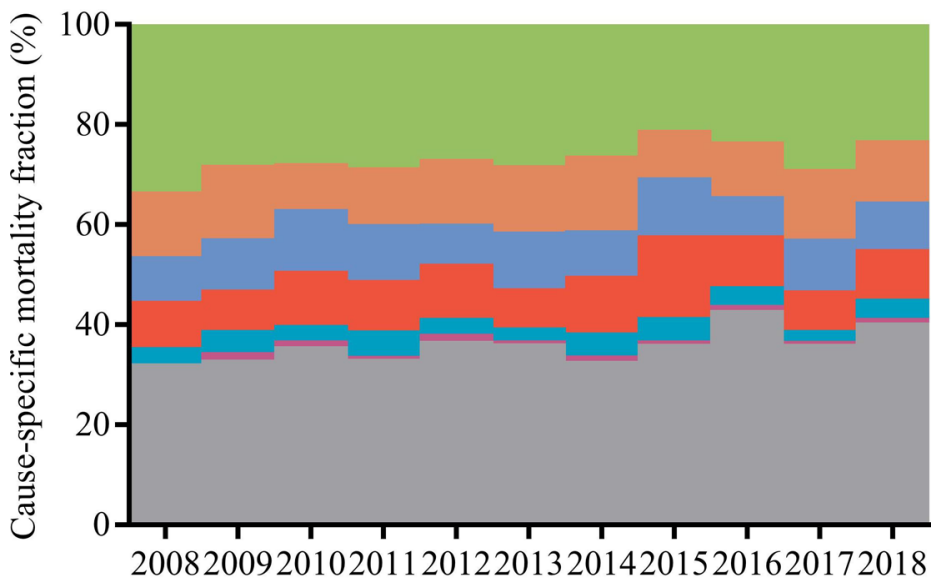

Obstetric haemorrhage

Amniotic fluid embolism

Pregnancy induced hypertension

Heart disease

Hepatopathy

Puerperal infection

Others

Year

Figure 4 Causes of maternal mortality in China, 2008-2018. Notes: data were extracted from the National Health Statistics Yearbooks in China. ${ }^{4}$ The proportion of puerperal infection in 2008 was included in other causes. 


\section{STRATEGIES}

To cope with these challenges, based on the previous evidence and country experience, the National Health Commission implemented a package of nationwide strategies in April 2016. These are called the Five Strategies for Maternal and Newborn Safety (FSMNS). They involve taking into account the natural progress of cases that ended in death, from low-risk pregnancy to high-risk pregnancy to critical pregnancy to death. The FSMNS consists of five components: (1) pregnancy risk screening and assessment strategy, (2) case-by-case management strategy for high-risk pregnancies, (3) referral and treatment strategy for critically ill pregnant women and newborns, (4) reporting strategy for maternal deaths and (5) accountability strategy (box 1 ). ${ }^{18}{ }^{19}$ Among the five strategies, screening for and assessment of high-risk pregnancies is the foundation of high-quality perinatal healthcare. All the pregnant women are screened and assessed by medical workers using uniform standards and procedures, all of whom have undergone unified training (figure 5). After risk assessment and classification, medical records of women are labelled with green (low risk), yellow (moderate risk), orange (high risk), red (highest risk) or purple (infectious disease) for tailored management. Pregnant women with different risk levels are transferred to designated medical institutions at different levels for further perinatal care and delivery (figure 5). Case-by-case management is implemented on pregnancies at higher level of risk (labelled with orange, red, and purple).

\section{ACTION AND IMPACT}

To better implement the FSMNS, China formulated a unified pregnancy risk screening form through multiple rounds of expert consensus. This form has already been used to standardise risk classification nationwide. Tailored care has been provided for pregnant women according to their established level of risk according to the FSMNS. Limited high-quality medical resources are prioritised for risker pregnancies to foster maternal and infant safety. In order to ensure quality, the National Health Commission organised expert panels to train, supervise and assess the implementation of the FSMNS and gave feedback to local leaders and practitioners. China also launched an online direct reporting information system on MMR surveillance at the individual level on 1 October $2017 .^{19}$ This is the first time robust collection of national MMR data at individual level. It has been performed to help decision makers make timely decisions using the latest data on maternal safety and to help officials improve areas that fail to meet the requirements for reducing the MMR. The accountability strategy also provides quality assurance regarding the implementation of FSMNS.

China has established an integrated maternal and child health service system, which combines functions of disease prevention and clinical treatment to foster the promotion of maternal and child health, especially
Box 1 Key points in the Five Strategies for Maternal and Newborn Safety

\section{Pregnancy risk screening and assessment}

Risk screening for pregnant women is carried out during the first visit to a healthcare facility. The secondary or tertiary healthcare facilities use midwifery services to conduct pregnancy risk assessments and classify pregnancy risk according to risk severity. Medical records are labelled with five colours of 'green (low risk), yellow (moderate risk), orange (high risk), red (highest risk), and purple (infectious disease)' to facilitate classification and management. Pregnant women labelled with yellow, orange, red, and purple are instructed to seek maternal health services and hospital delivery at secondary or tertiary health facilities.

\section{Case-by-case management of high-risk pregnancies}

Pregnant women with different risk levels are transferred to different types of maternal and child health $(\mathrm{MCH})$ institutions for perinatal care and delivery. Women at greater risk (orange, red and purple) are considered a key population and sent for case-by-case management to foster whole-process management, dynamic supervision and centralised treatment and to facilitate the screening, registration, reporting, management and treatment of every high-risk pregnant women.

\section{Critically ill pregnant women and newborn referral and treatment}

A nationwide network of critically ill pregnant women and a newborn referral and treatment system has been established. A multidisciplinary maternal and neonatal critical care expert group has been established in each county. Maternal and neonatal critical care centres are responsible for critical care in designated areas, and they keep the green channel (express referral networks) open for critical illness referrals.

\section{Reporting maternal deaths}

Statistical tables of orange and red labelled cases have been established to guide the effective management and treatment of high-risk pregnant women. China launched a direct reporting system for individual maternal deaths for the first time on 1 October 2017. Within two hours of a maternal death, health facilities are required to report each case to the designated county-level maternal and child healthcare institutions. After checking the situation, designated personnel located at the countylevel maternal and child healthcare institution should report detailed information of the death case using the national annual online reporting information system for maternal and child health before the 10th day of each month. After a review of maternal deaths, rectification measures should be implemented by related health facilities.

\section{Accountability}

Areas that have achieved outstanding progress in the reduction of the maternal mortality ratio (MMR) should summarise and promote these effective experiences in a timely manner and be praised by the National Health Commission. For areas with increasing MMR trends, a group of experts should be assigned to provide training to a targeted guidance in a timely manner. For areas that have not implemented the required measures to reduce the MMR, the National Health Commission will issue criticism by circulating a notice. If a hospital sees maternal deaths continuously, the director of the related hospital will be held accountable.

with respect to reducing MMR. It provides a systematic guarantee for the implementation of the FSMNS. The establishment of maternal and child health systems has 


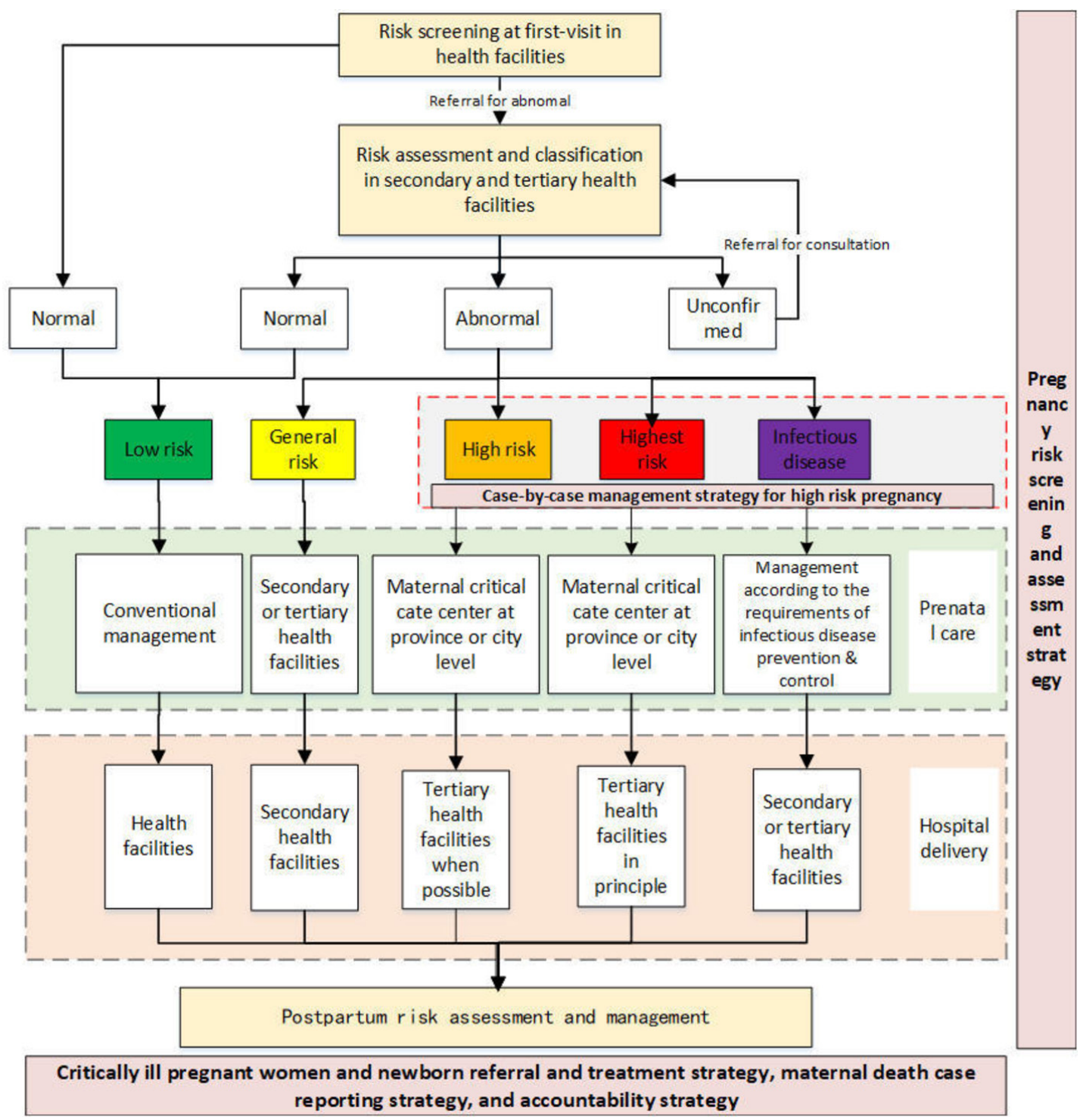

Figure 5 Risk assessment and management procedure of pregnant women in China.

also been improved during the implementation of the FSMNS. To facilitate the use of the FSMNS, the Chinese government invested 8.48 billion RMB on the support of infrastructure construction of maternal and child healthcare institutions nationwide. As of 2018, China had 3080 maternal and child healthcare institutions, 0.64 million practitioners, 807 obstetric and gynaecological hospitals, 129 children's hospitals, 400 million outpatient visits annually, 13.79 million inpatient visits annually and 0.34 million beds. ${ }^{20}$

After the implementation of the FSMNS, as of April 2018, pregnancy risk screening and assessment strategy was carried out in $97.8 \%$ of China's counties, and the case-by-case management strategy for high-risk pregnancy was carried out in $97.9 \%$ of all the counties. ${ }^{20} \mathrm{~A}$ total of 3364 maternal critical care centres and 3055 neonatal critical care centres have been established in China. ${ }^{20} \mathrm{~A}$ nationwide network of critically ill pregnant women and newborn referral and treatment has been established. A direct reporting mechanism for maternal deaths has also been established nationwide. ${ }^{20}$ The implementation of the FSMNS has prevented a rebound in the MMR in China in the era of the two-child policy, and reduced MMR related to the FSMNS was estimated 2.64 (95\% CI 1.87 to 3.41) per 100000 live births in 2018 (online supplementary file 1). The MMR in China has declined by $21.1 \%$, from 23.2 per 100000 live births in 2013 to 18.3 per 100000 live births in $2018 .{ }^{4}$

\section{CONCLUSION}

China should continue using the FSMNS, strengthen screening and management of high-risk pregnancies and increase investment in regions with relatively limited medical resources to ensure that all regions of the country reach the targets in the SDGs and Healthy China by 2030. Integration of disconnected national programmes and information systems related to maternal and child health is also needed to promote better management 
on high-risk pregnancies and continue reduction in the MMR in the future.

Acknowledgements We would like to thank the BMJ editor Dr Daoxin Yin for her suggestions on the structure of the paper.

Contributors JL, LS, GQ and ML developed the structure of the paper. All the authors contributed to the initial draft. All the authors worked on and approved the final version. $\mathrm{ML}$ and $\mathrm{GQ}$ contributed equally to this article and are the guarantors.

Funding This article is funded by the National Natural Science Foundation (81703240; 71874003).

Competing interests None declared.

Patient consent for publication Not required.

Provenance and peer review Not commissioned; externally peer reviewed.

Data availability statement № additional data are available.

Open access This is an open access article distributed in accordance with the Creative Commons Attribution Non Commercial (CC BY-NC 4.0) license, which permits others to distribute, remix, adapt, build upon this work non-commercially, and license their derivative works on different terms, provided the original work is properly cited, appropriate credit is given, any changes made indicated, and the use is non-commercial. See: http://creativecommons.org/licenses/by-nc/4.0/.

ORCID iD

Min Liu http://orcid.org/0000-0002-5059-3743

\section{REFERENCES}

1 GBD 2015 Maternal Mortality Collaborators. Global, regional, and national levels of maternal mortality, 1990-2015: a systematic analysis for the global burden of disease study 2015. Lancet 2016;388:1775-812.

2 Ministry of Foreign Affairs People's Republic of China,, United Nations System in China. Report on China's implementation of the millennium development goals (2000-2015), 2015. Available: http:// www.cn.undp.org/content/china/zh/home/library/mdg/mdgs-report2015-/

3 Liang J, Li X, Kang C, et al. Maternal mortality ratios in 2852 Chinese counties, 1996-2015, and achievement of millennium development goal 5 in China: a subnational analysis of the global burden of disease study 2016. Lancet 2019;393:241-52.

4 National Health Commission of the People's Republic of China. The 2019 National health statistics Yearbook. Beijing: China Union Medical University Press, 2019.
5 The State Council of the People's Republic of China. The healthy China 2030 plan, 2016. Available: http://www.gov.cn/zhengce/201610/25/content_5124174.htm

6 National office of National Maternal and Child Health Surveillance System, National office of National Maternal and Child Health Annual Reporting System. National Maternal and Child Health Surveillance and Annual Reporting System Report (volume 1\&2), 2018. Available: http://www.mchscn.org/Article_Class2.asp?ClassID $=47$

7 WHO. World health statistics 2019: monitoring health for the SDGs, sustainable development goals, 2019. Available: https://apps.who. int/iris/bitstream/handle/10665/324835/9789241565707-eng.pdf [Accessed 27 Sep 2019].

8 The State Council of the People's Republic of China. Decision on implementing universal Two-Child policy reform and Perfecting family planning service management, 2015. Available: http://www. gov.cn/gongbao/content/2016/content_5033853.htm

9 Lee S, Ayers S, Holden D. Risk perception of women during high risk pregnancy: a systematic review. Health Risk Soc 2012;14:511-31.

10 Zelop CM, Einav S, Mhyre JM, et al. Cardiac arrest during pregnancy: ongoing clinical conundrum. Am J Obstet Gynecol 2018:219:52-61.

11 Carroll AE. Why is us maternal mortality rising? JAMA 2017;318:321.

12 D'Alton ME, Main EK, Menard MK, et al. The National partnership for maternal safety. Obstet Gynecol 2014;123:973-7.

13 Sauer MV. Reproduction at an advanced maternal age and maternal health. Fertil Steril 2015:103:1136-43.

14 Liang J, Mu Y, Li X, et al. Relaxation of the one child policy and trends in caesarean section rates and birth outcomes in China between 2012 and 2016: observational study of nearly seven million health facility births. BMJ 2018;360:k817.

15 Lumbiganon P, Laopaiboon M, Gülmezoglu AM, et al. Method of delivery and pregnancy outcomes in Asia: the who global survey on maternal and perinatal health 2007-08. Lancet 2010;375:490-9.

16 Zhang $\mathrm{H}-\mathrm{X}$, Zhao Y-Y, Wang Y-Q. Analysis of the characteristics of pregnancy and delivery before and after implementation of the Twochild policy. Chin Med J 2018;131:37-42.

17 Zeng C, Yang M, Ding Y, et al. Placenta accreta spectrum disorder trends in the context of the universal two-child policy in China and the risk of hysterectomy. Int J Gynecol Obstet 2018;140:312-8.

18 National Health Commission. Specification of the effective management and clinical treatment of pregnant women with advanced maternal age, 2016. Available: http://www.nhfpc.gov.cn/ fys/gzbs/201604/73f2853f2c634df7bb1d577d9de845cc.shtml

19 National Health Commission. Notice on strengthening maternal and child safety service, 2017. Available: http://www.gov.cn/gongbao/ content/2018/content_5265003.htm

20 National Health Commission. Report on the maternal and child health care development (2019), 2019. Available: http://www.nhc. gov.cn/fys/s7901/201905/bbd8e2134a7e47958c5c9ef032e1dfa2. shtm 